\title{
Las mujeres en situación de violencia y acceso a la justicia en el Noroeste Argentino. Del diagnóstico a la acción
}

Women in situations of violence and the access to justice in the Argentine Northwest. From diagnosis to action

Fernanda Maria Marchese ${ }^{1}$, Maria Fernanda Rotondo ${ }^{2} y$ Ariadna Soledad Espíndola Pinela ${ }^{3}$

\section{Resumen}

Este trabajo propone no solo investigar cuales son los obstáculos que encuentran las mujeres, cis, trans, lesbianas, pertenecientes a diferentes sectores: mujeres indígenascampesinas, migrantes y defensoras de derechos humanos, frente al acceso a la justicia, sino también proponer y llevar adelante una solución a los mismos.

Palabras clave: Mujeres, violencia de género, acceso a la justicia, derechos humanos

\section{Abstract}

This work proposes not only to investigate what are the obstacles, that women, cis, trans, lesbians, belonging to different sectors: indigenous-peasant women, migrants and human rights defenders, face against access to justice, but also to propose and carry out a solution to them.

Keywords: Women, gender violence, access to justice, human rights

\footnotetext{
Recibido: 31 de marzo de 2021 Aceptado: 30 de junio de 2021 Publicado: 8 de julio de 2021

${ }^{1}$ Profesora en Ciencias de la Educación (UNT). Directora Ejecutiva de la Fundación ANDHES. Profesora de la Cátedra de Política Educativa de la Facultad de Filosofía y Letras y Asesora pedagógica de la Escuela y Liceo Vocacional Sarmiento (UNT). Correo electrónico: fermarchese85@gmail.com ID https://orcid.org/00000002-6422-3786

${ }^{2}$ Estudiante avanzada de la Lic. en Ciencias de la Comunicación, Universidad Nacional de Tucumán (UNT). Integrante del Instituto de Historia y Pensamiento Argentino (IHPA) de la Facultad de Filosofía y Letras (UNT). Coordinadora del Área de Género y Derechos Humanos de la Fundación ANDHES sede Tucumán. Correo electrónico: mariferotondo@gmail.com ID https://orcid.org/0000-0003-2190-9494

${ }^{3}$ Estudiante avanzada de la Lic. en Psicopedagogía de la Universidad Católica de Santiago del Estero sede San Salvador (UCSE-DASS). Diplomada en Violencia de Género, Derechos y Movimientos de Mujeres (UNJu). Coordinadora del Área de Género y Derechos Humanos de la Fundación ANDHES sede Jujuy. Correo electrónico: arisoles32@gmail.com ID https://orcid.org/0000-0001-7459-4436
} 


\section{Introducción}

La presente publicación, tiene por finalidad dar a conocer el proyecto de investigación llevado a cabo por ANDHES (Abogados y abogadas por los derechos humanos y estudios sociales), junto con CLADEM (Comité de Latinoamérica y el Caribe para la Defensa de los Derechos de la Mujer), en las provincias de Jujuy y Tucumán, desde el año 2020, "Las mujeres en situación de violencia y acceso a la justicia en el Noroeste Argentino. Del diagnóstico a la acción”.

Cabe aclarar, que en lo que a este proyecto respecta, hablar de mujeres es hablar de mujeres Cis, Trans, Lesbianas, de Pueblos Indígenas, Campesinas y Activistas.

Este proyecto financiado por el Fondo Fiduciario de la ONU tiene como finalidad generar buenas prácticas en materia de Derechos Humanos, democratizando conocimientos, para poder visibilizar y sensibilizar sobre las problemáticas que atraviesan las mujeres en el acceso a la justicia.

Andhes desde sus inicios, hace más de quince años, lleva adelante procesos de fortalecimiento jurídico con los colectivos con los cuales trabaja (niños, niñas y adolescentes, pueblos indígenas, migrantes), entre ellos las mujeres CTL. En esta agenda de trabajo tiene como objetivo avanzar en la erradicación de todas las formas de violencia hacia las mujeres, niñas, adolescentes, lesbianas, trans, travestis, facilitar el derecho como una herramienta al alcance de estos colectivos, para incidir en la formulación de políticas públicas que garanticen la igualdad en el campo laboral, económico y político y la posibilidad de gozar plenamente de los derechos sexuales y reproductivos vigentes.

El conocimiento de años de trabajo en conjunto con mujeres cis, trans y lesbianas (CTL) de ambas organizaciones nos permite comprender la importancia de contar con guías y protocolos de acción que sean claros para ser ejecutados durante los años que corresponden a los objetivos del proyecto.

El plan de acción cuenta con tres años de duración.

El primer año (2020) desarrollamos investigación participativa, que es uno de los componentes más importantes del proyecto, ya que se busca detectar los obstáculos que actúan como barreras en el acceso a la justicia de las mujeres CTL en situaciones de violencia en las provincias de Jujuy y Tucumán (Argentina).

El segundo año (2021), realizaremos talleres para aumentar las capacidades técnico-jurídicas de las mujeres cis, trans, lesbianas, trabajadoras; migrantes, activistas políticas e indígenas, en la defensa de sus derechos.

El tercer año (2022) las beneficiarias podrán poner en práctica lo aprendido y así facilitarán el acceso a la justicia en sus comunidades con la creación de centros comunitarios de defensa de derechos (CDD). 
La presente publicación dará a conocer, sobre todo, lo relativo al primer año del proyecto, que sufrió el impacto del contexto de pandemia por COVID-19 afectando sobre todo a los sectores más vulnerables.

\section{Objetivos}

El objetivo general de este proyecto es identificar los obstáculos de acceso a la justicia ante los que se encuentran las mujeres CTL, para así poder erradicarlos.

Durante el primer año 2020, se pretendió producir datos sobre los obstáculos para el acceso a la justicia y fortalecer las capacidades de las referentes para recolectar y analizar datos de su contexto. Además, se llevó adelante la construcción de una herramienta de mapeo susceptible de ser actualizada los años posteriores del proyecto y accesible para el relevamiento de información territorial.

Los datos fueron obtenidos a través de entrevistas y encuestas escritas. En las mismas, las beneficiarias del proyecto a través de los relatos de sus historias de vida dieron a conocer sus inquietudes, desafíos y experiencias personales en relación a las instituciones estatales a la hora de afrontar casos de violencia.

Por otro lado, se realizaron entrevistas con representantes de distintos poderes del Estado provincial de Jujuy y Tucumán, y con representantes de los distintos poderes del Estado Nacional. A su vez, enviamos pedidos de información pública a cada una de las reparticiones provinciales, a los fines de obtener información clara y precisa, que no pudo obtenerse en las entrevistas. Sin embargo, cabe aclarar que hasta la fecha, no tuvimos respuestas de algunas instituciones del Estado pero continuaremos fortaleciendo los intercambios oportunamente en el tiempo que falta de desarrollo del proyecto .

\section{Contexto}

De acuerdo a la Convención de Belém Do Pará (1996), el análisis de contexto debe hacerse a través de tres indicadores (estructurales, de proceso y de resultado).

Los indicadores estructurales nos permiten, identificar la normativa legal con la que cuenta nuestro país, para así reflexionar sobre su aplicación efectiva.

Los indicadores de proceso permiten verificar y vigilar la aplicación de las políticas públicas.

Los indicadores de resultado permiten el análisis del impacto real de aquellas, políticas públicas, en este caso, en la vida de las mujeres, de manera cuantitativa y cualitativa.

De esta forma, entramos en la cuenta de que Argentina cuenta con un amplio marco legal, adecuado a los estándares internacionales de derechos humanos. Esto es así, porque, nuestro país ratificó múltiples tratados internacionales a los cuales se les 
otorgó jerarquía constitucional (art. 75 inc $22 \mathrm{CN}$ ), como por ejemplo: "Convención De Belem Do Para", para prevenir, sancionar y erradicar la violencia contra la mujer, la Convención Trata de personas, Ley 25.632 del año 2002, Convención sobre la eliminación de todas las formas de discriminación contra la Mujer (CEDAW), Ley 23.179 del año 1985, la Convención Americana sobre Derechos Humanos, ratificada por Ley N²3.054 del año 1984, la Declaración Universal de los Derechos Humanos, Pacto Internacional de Derechos Civiles y Políticos, Principios de Yogyakarta (con 37 principios y 18 recomendaciones), entre otros.

A nivel nacional en lo que respecta a normas de interés público de aplicación en todo el territorio de la nación, podemos mencionar la aprobación de la Ley 26.485 (2009) de Protección Integral a las Mujeres, que significó un cambio y avance en la materia.

En las provincias, en razón de la autonomía provincial, se aprobaron normas de adhesión, considerando las particularidades de cada contexto.

En cada localidad, coexisten múltiples protocolos de actuación, aprobados por distintos ministerios, que muchas veces no son conocidos ni aplicados por las autoridades a los que están destinados, lo que deja en evidencia la dificultad en la articulación dentro de cada ámbito.

Nuestro proyecto de investigación se centró particularmente como ya se mencionó, en dos provincias del norte argentino, Tucumán y Jujuy.

Considerando que Argentina es un país federal, para analizar el contexto de cada una de nuestras provincias, resulta necesario repasar el contexto nacional.

\subsection{Avances durante el 2020}

\subsubsection{A Nivel Nacional}

Como es de público conocimiento, en razón de la pandemia por covid-19, el gobierno nacional en marzo de 2020 dispuso el Aislamiento Social Preventivo y Obligatorio (ASPO) que, si bien fue la medida recomendada por la OMS para atacar la pandemia del Coronavirus, no podemos dejar de lado la forma en la que el encierro impactó en las mujeres y en las personas LGBTIQP+.

$\mathrm{Al}$ ser los mencionados, grupos vulnerables, vieron incrementados los múltiples hechos de violencia patriarcal que atraviesan cotidianamente. Razón por la cual, como una medida paliativa de ello se dictó el Decreto N²97 del 19 de marzo de 2020, en este el Gobierno argentino dispuso que las mujeres y personas LGBTIQP+ estaban autorizadas a abandonar sus domicilios "solas o junto a sus hijos e hijas" para denunciar, pedir auxilio, asistencia o protección en razón de la situación de violencia que se encuentren transitando, considerando éstos casos "supuestos de causa mayor". 
Asimismo, se reforzaron los canales de atención de la Línea 144, sumando recursos humanos y tecnologías para los equipos de atención, además habilitó otros medios de contacto como WhatsApp, correo electrónico y una aplicación para celulares.

Desde el Ministerio de las Mujeres, Géneros y Diversidad de la Nación, creado con la asunción del nuevo gobierno en el año 2019, se comprometieron a fortalecer las casas de acogida, incluyendo establecimientos de sindicatos y universidades para alojamiento de mujeres en situación de violencia que necesitaran abandonar sus hogares durante el periodo de aislamiento. Además de gestionar los recursos necesarios para las casas de acogida, a través de canales específicos para la vinculación directa con gobiernos provinciales, locales, y con las organizaciones de la sociedad civil, comunitarias y territoriales.

Específicamente para personas trans, el Gobierno Nacional gestionó la incorporación a los programas sociales del Ministerios de Desarrollo Social de la Nación, realizó recomendaciones para garantizar su acceso a la salud, poniendo en marcha el plan Potenciar Trabajo en todo el país.

El mismo ministerio, lanzó el Registro Nacional de Trabajadores y Trabajadoras de la Economía Popular que incluye en las categorías posibles "trabajadores sexuales" con el fin de habilitar el acceso a herramientas y programas sociales.

Asimismo, se crearon módulos alimentarios para personas trans travestis: se entregaron 1500 módulos alimentarios desde el MMGyD. Y a través del decreto nacional $\mathrm{N}^{\circ} 310 / 20$, se otorgó el IFE: Ingreso familiar de emergencia como prestación monetaria no contributiva de $\$ 10.000$ de carácter excepcional destinada a compensar la pérdida o grave disminución de ingresos de personas afectadas por la situación de emergencia sanitaria.

En lo que refiere al sistema de justicia, el Poder Judicial de la Nación también tomó algunas medidas, como el hecho de prever formas alternativas para la recepción de denuncias por violencia de género, a través de Whatsapp, llamada telefónica u otros medios. En este sentido la Corte Suprema de Justicia de la Nación autorizó el libramiento electrónico de las órdenes de pago en concepto de alimentos mediante la Acordada No 9/2020.

Aunque no todas las provincias hayan tomado estas medidas, la mayoría de las provincias estableció la prórroga automática de medidas de protección en casos de violencia de género por un período determinado y/o hasta que finalice el aislamiento social, preventivo y obligatorio, dependiendo esta decisión de la Justicia de cada Provincia. Por su parte, la provincia de Jujuy, adhirió al decreto presidencial que declara al servicio de justicia en lo que respecta a asistencia a víctimas de violencia de género como un servicio esencial. 


\subsubsection{Tucumán}

- Se sancionó la adhesión a la Ley Micaela, estableciendo la capacitación obligatoria en perspectiva género para quienes integran los poderes del Estado.

-El Cupo Laboral Trans en el Poder Judicial y en la Municipalidad de San Miguel de Tucumán; que fueron el resultado de la exigencia de las organizaciones de mujeres y de personas LGBTIQP+. Esto se enmarca, en encontrar mayor legitimidad en sus peticiones dada la existencia de instituciones nacionales que instan al cumplimiento efectivo de los derechos humanos.

- Los mecanismos de denuncias existentes ya se encontraban en un proceso de ampliación con anterioridad, ya que se instalaron Oficinas de Violencia doméstica en distintos puntos de la provincia (cinco en total: Capital, Banda del Río Salí, Trancas, Concepción y Monteros), y que se cuenta ya con algunas comisarías y otras oficinas especializadas distribuidas territorialmente.

Sin embargo, a pesar de ello, las medidas adoptadas no fueron suficientes aumentando considerablemente los índices de femicidios en ambas provincias. Desde que inició el ASPO, ocurrieron 19 femicidios en el año 2020, superando así la cifra de 2019 (11 femicidios en total de enero a diciembre), convirtiendo a Tucuman en la tercera provincia con más casos de asesinatos de mujeres en todo el país durante este año.

Según la Red Ni Una Menos Tucumán el aumento de llamadas fue de un 200\% en oficinas como el Observatorio de la Mujer y la Oficina de Violencia Doméstica (OVD) de la provincia. De la misma forma, la Secretaria Provincial de la Mujer María del Carmen Carrillo, informó que en las últimas semanas de abril, recibieron 600 llamadas a través de los números de emergencia. Esto motivó a que, ante la falta de refugios para víctimas de violencia de género se rehabilitarán aquellos que habían dejado de funcionar desde que inició la cuarentena. faltaría la cita tipo Autor corporativo, informe gubernamental

En relación a mujeres trans, ya en el año 2013, Celeste fue detenida arbitrariamente por la policía de la provincia, privada de su libertad en condiciones insalubres y sometida a violencia sexual y psicológica . También en Tucumán, pero en el año 2012, Ayelén Gómez fue detenida arbitrariamente junto al chofer del taxi que la trasladaba luego de una fiesta. En ese episodio sufrió abusos sexuales por los policías de la comisaría 2 da de la capital provincial y fue amenazada de muerte. Finalmente, en el año 2017 fue asesinada. 


\subsubsection{Jujuy}

En Jujuy, una ola de femicidios sucedidos en aproximadamente tres semanas entre fines de septiembre de 2020 y principios de Octubre de 2020, dio lugar a fuertes movilizaciones que a su vez ocasionaron la reacción estatal.

En lo que refiere a los dispositivos de atención en caso de violencia de género, en Jujuy el Consejo Provincial de la Mujer contó durante el ASPO con 15 dispositivos distribuidos en toda la provincia, a pesar de ello fue notable la falta de personal trabajando dentro de cada uno. Se amplió la cantidad de líneas de comunicación en diferentes localidades de la provincia y se habilitó un centro de diversidad en la capital provincial. El Ministerio Público de la Acusación habilitó canales de denuncias en casos de violencia de género un 0800 y mail del centro de asistencia a la víctima, además de los números de guardia de los fiscales. Además, se nombraron y sumaron dos fiscales para cubrir los cargos de fiscales en violencia de género. Sobre las estadísticas, este organismo en una transmisión del Comité Operativo de Emergencia se informó que se registraron: 11 casos de muertes en contexto de violencia de género durante el 2020, 8 de las cuales se investigan como femicidios.

Sin embargo, no se cuenta con información certera sobre el funcionamiento de dicho observatorio, ni hay acceso público a las estadísticas ni comunicaciones oficiales con esta información, sino solo un video del COE, el $\mathrm{N}^{\circ} 205$, en el que pasaron placas con los datos mencionados.

A pesar de no contar con datos oficiales del Estado, de los datos arrojados por una investigación periodística del diario "Jujuy es noticia”, surge que durante el 2020 se registraron 12 casos de femicidio, y sólo 10 están siendo investigados como tales.

El denominador común, de acuerdo a los reclamos públicos de familiares y amigos de las víctimas es la desidia e inacción del Estado provincial, el incumplimiento de protocolos, el no acceso a la justicia y la falta de debida diligencia.

El resultado de los reclamos y movilizaciones sociales encabezados no solo por asociaciones civiles, sino también por parientes, y cercanos de las víctimas, y otros militantes independientes fue la aprobación en sesión especial de la Ley de Emergencia por Violencia de Género, por la legislatura provincial.

Del mismo modo, el Superior Tribunal de Justicia de la provincia de Jujuy declaró la emergencia en Violencia de Género en la justicia, mediante acordada $114 / 2020$ y ordenó la creación de tres nuevos juzgados en violencia de género en las localidades de Perico, Humahuaca y Libertador General San Martin. Sin embargo, hasta la fecha no cuentan con asiento en dichas localidades, por no poseer un lugar físico, más aún los y las funcionarias que hoy ocupan el cargo de jueces en el fuero de violencia, no lo hacen por designación oficial, sino simplemente por habilitación y subrogancia. En otras palabras, la judicatura no está compuesta por especialistas en 
Violencia de Género, ni abocaron sus carreras a la complejidad de estas cuestiones, incumpliendo con la Ley Micaela y su adhesión.

Sin dejar de lado la situación de las mujeres trans, en Jujuy, un caso conocido fue el de Delfina Díaz, una chica trans de 19 años que denunció que el 13 de abril de 2020 fue detenida por cuatro policías, tres varones y una mujer, en la ciudad de Libertador General San Martín. La detención fue supuestamente en el marco de las medidas de prohibición de circulación, pero los efectivos la trasladaron a un descampado donde fue abusada sexualmente y amenazada.

Por otro lado, Jujuy cuenta con una diferencia fundamental en relación a las demás provincias del país, ya que, su población está conformada por un gran porcentaje de mujeres indígenas, rurales y migrantes. El entramado social es complejo porque construyen una trama de convivencia con pocas marcas diferenciales pero que, al mismo tiempo, mantiene un fuerte deseo de diferenciarse; el cual se plasma en las perspectivas, en materia de acceso a derechos de mujeres. Muy contrariamente, lo que refiere a políticas públicas, estas son pensadas desde una lógica colonial y hegemónica.

Como ejemplo más concreto de este obstáculo de acceso a la justicia en la provincia de Jujuy, en el cual el Estado ha fallado en su deber de debida diligencia, revelando una estructura en la administración de justicia carente de una perspectiva integral e interseccional sobre la violencia contra las mujeres indígenas-campesinas, citamos el caso de Claudia Puma Espíritu, mujer migrante, indígena y quechua hablante acusada de filicidio quien estuvo presa en la Unidad 3 del Penal de Mujeres desde el año 2017 y privada de ejercer su derecho de defensa. Este caso es relevante no solo por las circunstancias del hecho imputado a la mujer y su investigación, sino también por las vulneraciones atravesadas en su situación de encierro, donde sufrió abusos; tras cursar un proceso lleno de irregularidades donde no se le proveyó de un/a intérprete, ya que Claudia no comprendía el idioma español y con una suspensión de juicio a prueba por más de tres años. Fue finalmente absuelta de la causa, en noviembre de 2020, ya que no existían pruebas suficientes que la acusaran y quedó manifiesto que fue víctima de una justicia sin perspectiva intercultural, interseccional y de género, que obstaculiza su derecho de defensa.

Aún más, en el segundo semestre del año 2020 se produjeron dos hechos relevantes, en diferentes comunidades indígenas y campesinas de la provincia de Jujuy. Por un lado, en octubre del 2020, se originó un proceso de desalojo, ordenado por la justicia jujeña, seguido por una represión policial, sufrida por la comunidad indigena Tusca Pacha de los Alisos, ubicada en la Ciudad de Palpalá de la Provincia de Jujuy. El segundo hecho data desde agosto del 2020, donde las mujeres de Pueblo de Tilian, ubicado en la localidad de Volcán, Departamento Tumbaya de la Provincia 
de Jujuy; comenzaron un proceso de denuncias por violencia de género al terrateniente Gustavo Bárcena, consiguiendo por primera vez una medida cautelar de protección de persona en el marco de un conflicto territorial.

En base a todo este contexto regional mencionado, podemos observar que comprende imaginarios sociales que alimentan los prejuicios imperantes reflejados en actos y discursos hegemónicos segregacionistas, como así también, se sigue evidenciando acciones dentro del Estado y la justicia que obstaculizan el acceso a la misma.

Es por ello, que nos proponemos cuestionar esos mandatos a partir de prácticas de desnaturalización y visibilización de las problemáticas vinculadas a la temática, desde este proceso de investigación acción-participativa.

\subsubsection{Análisis del Contexto}

En base al análisis de estos datos, podemos decir que a pesar de las normativas vigentes, y de las medidas tomadas por el gobierno nacional en materia de género durante el contexto de ASPO; las mujeres cis, trans y lesbianas (CTL) en Argentina, siguen estando en una grave situación de vulneración y marginalidad. Además de la persistencia de estereotipos sexistas y discriminatorios hay una marcada ausencia de articulación entre los poderes ejecutivo, legislativo y judicial dentro de cada provincia, y más aún hay desarticulación entre el gobierno nacional y los gobiernos provinciales.

Esto queda demostrado, por ejemplo, que aunque en el Estado Nacional es obligatoria la capacitación en perspectiva de género de sus agentes, a partir de la sanción de la Ley Micaela (2019), hubo una gran resistencia por parte de los gobiernos provinciales a la aplicación de las normativas que protegen los derechos de las mujeres CTL.

La falta de articulación de políticas públicas nacionales y provinciales (las cuales son muy diversas) sigue siendo un desafío no superado, agravado por la pandemia de COVID-19, que como resultado impactó en los sectores más vulnerables, obstaculizando la aplicación de los planes de gobierno tendientes a erradicar las asimetrías existentes.

En este contexto, es claro que las políticas de gobierno no fueron suficientes, ya que, según el informe del Observatorio de las Violencias de Género "Ahora Que Sí Nos Ven" se registraron en Argentina, desde el 1 de enero al 30 de diciembre del 2020, 298 femicidios: 25 femicidios sucedieron durante el mes de diciembre; es decir 1 femicidio cada 29 horas en el periodo nombrado. A esto se suman 5 travesticidios/transfemicidios criminales y 103 son los travesticidios/transfemicidios sociales durante el año; lo cual pone de manifiesto que aún queda mucho por hacer. 
En Tucumán y Jujuy la violencia contra las mujeres y los femicidios incrementaron considerablemente sumando 19 femicidios en Tucumán y 12 en la provincia de Jujuy en el año 2020.

Según éste informe del Observatorio de las Violencias de Género "Ahora que si nos ven”, el 65,5\% fue cometido por las parejas y exparejas de las víctimas, y el 65,1\% ocurrió en la vivienda de la víctima, y al menos 256 niños y adolescentes perdieron a sus madres como consecuencia de la violencia machista en 2020, muchos de ellos incluso, presenciaron el hecho violento y/o también convivían con el femicida. falta la cita

En lo que respecta a los derechos sexuales y reproductivos y no reproductivos de las mujeres y niñas, particularmente al acceso a las Interrupciones Legales del Embarazo (ILE), en 2019 los gobiernos provinciales de Tucumán y Jujuy obstaculizaron abortos no punibles de niñas menores de 13 años, (caso Lucía y Niña DAM). En la provincia de Tucumán, la situación se agrava al no estar adheridas a leyes nacionales de Salud Sexual y Reproductiva y de Educación Sexual Integral (ESI).

Las mujeres CTL se encuentran desamparadas ante la connivencia explícita entre los poderes del Estado y sectores que se oponen al ejercicio de sus derechos. Durante el 2018, mientras se discutía en el Congreso de la Nación la legalización de la interrupción voluntaria del embarazo (IVE), la legislatura de Tucumán se declaró “provida”. En 2017, Tucumán fue noticia por el Caso Belén, joven que estuvo presa casi dos años por un aborto espontáneo. Además, tiene una alta tasa de embarazo adolescente (5000 nacimientos de adolescentes de entre 15 y 19 años).

Como resultado de la lucha de los movimientos feministas y transfeministas, por la efectivización de derechos reproductivos y no reproductivos de las personas gestantes, llevó a instalar nuevamente el debate sobre la interrupción voluntaria del embarazo en el Congreso de la Nación, proyecto de ley que fue finalmente sancionado el 29 de diciembre de 2020. Este fue sin lugar a dudas el debate que más problematizó la vulneración de los derechos sexuales reproductivos y no reproductivos. Además, sacó de la clandestinidad las malas prácticas llevadas adelante por los efectores del Estado en lo que respecta al acceso a la interrupción legal del embarazo. Finalmente, la ley entró en vigencia con el $\mathrm{N}^{\circ} 27.610$ y fue fuertemente atacada por el grupo denominado "provida", quienes luego de su aprobación anunciaron que insistirán por la vía judicial para impedir su aplicación. En consecuencia, la justicia de Salta habilitó la feria judicial para rechazar un recurso colectivo interpuesto por este sector, queda ahora la tarea de velar para que las instituciones garanticen su cumplimiento efectivo.

Con respecto a las mujeres travestis-trans, su promedio de vida varía entre los 35 y 40 años a diferencia de la expectativa general de la región que es de 75 años; esto 
a raíz de las condiciones en las cuales se desarrolla su existencia. La criminalización y la clandestinidad de las identidades trans en todas las provincias del país representa también un gran obstáculo para el acceso a sus derechos. Es una constante para las mujeres trans el abuso de autoridad y la violencia policial. Los agentes de seguridad las tratan sin respeto por su identidad y utilizan las contravenciones de manera extorsiva para acusarlas de ejercer el trabajo sexual por el solo hecho de estar en la vía pública. Algunos casos que resonaron: Diana Sacayán (referente del Movimiento Antidiscriminatorio de Liberación) fue golpeada y arrestada sin causa en 2015 por la policía de la Ciudad de Buenos Aires, el hecho fue denunciado y luego fue asesinada en su casa.

En cuanto a las mujeres lesbianas, la falta de mayor información respecto a su situación particular da cuenta de la falta de interés por parte del Estado a sus situaciones particulares de violencia. Pese a este contexto particularmente encarnizado en el norte del país, el movimiento feminista logró visibilizar e instalar la lucha contra las violencias en diferentes sectores de la sociedad en los últimos años. Por su parte, a pesar del rechazo de los sectores conservadores que se encuentran en el poder, existen funcionarios/as y otros/as agentes estatales comprometidos/as con la problemática y con interés en que los marcos legales nacionales funcionen adecuadamente en sus jurisdicciones.

Por otra parte, las mujeres indígenas y migrantes son víctimas también de la desigualdad estructural que se refuerza y profundiza. Una medida regresiva en materia de protección y acceso a derechos en el año 2017, por medio del Decreto de Necesidad y Urgencia (DNU 70/2017), se derogó parte sustancial de la Ley de Migraciones, retrocediendo en la garantía de los derechos de la población migrante. Las modificaciones introducidas por este decreto violan las garantías de debido proceso y el acceso a la justicia en el marco de los procedimientos de expulsión y detención por razones migratorias. Modificaciones que impactan diferencialmente en las mujeres migrantes.

En lo que respecta a la normativa que atiende la protección integral de las mujeres, la ley 26.485, sobre las mujeres indígenas, establece en su Art. 3 que su objeto es: sensibilizar, prevenir, sancionar y erradicar la discriminación y la violencia contra las mujeres en cualquiera de sus manifestaciones y ámbitos; manteniendo una protección generalizada sin considerar particularidades para abordar problemas de mujeres indígenas, afrodescendientes, migrantes, etc. falta la cita

Por otro lado, con la reforma de la Constitución Nacional Argentina de 1994, se incorporó en el artículo 75, inciso 17, la siguiente obligación para el Congreso de la Nación: reconocer la preexistencia étnica y cultural de los pueblos indígenas argentinos. Garantizar el respeto a su identidad y el derecho a una educación bilingüe 
e intercultural; reconocer la personería jurídica de sus comunidades, y la posesión y propiedad comunitarias de las tierras que tradicionalmente ocupan; y regular la entrega de otras aptas y suficientes para el desarrollo humano y asegurar su participación en la gestión referida a sus recursos naturales y a los demás intereses que los afecten. falta la cita

La ley 26.160 declaró la emergencia en materia de posesión y propiedad de las tierras que tradicionalmente ocupan las comunidades indígenas originarias del país, tema no menor ya que las tierras ancestrales conforman un elemento esencial de la existencia y cultura de las mujeres indígenas. En el plano internacional, Argentina suscribió el Convenio 169 de la O.I.T. que avanza en un paradigma intercultural y reconoce los derechos humanos de los pueblos indígenas. Sin embargo, el no reconocimiento a sus tierras sigue siendo un grave problema que enfrentan las Comunidades, enfrentándose constantemente a vulneraciones a sus derechos al territorio, mediante procesos judiciales con órdenes judiciales y extrajudiciales de desalojo arbitrarias. falta cita

\section{El acceso a la justicia y sus obstáculos}

Como todo derecho, el acceso a la justicia requiere un sistema de garantías que posibilite su pleno ejercicio. Esto supone la obligación del Estado de crear las condiciones jurídicas y materiales que garanticen su vigencia en condiciones de igualdad (Birgin, 2012). Sin embargo, observamos que existen factores que limitan su real acceso, traducidos en obstáculos que pueden ser procesales (vinculados a las instituciones públicas); materiales (condiciones sociales, económicas, culturales, etc. del grupo); y/o de orden simbólico (prácticas sociales que naturalizan la desigualdad y la discriminación).

\subsection{Las mujeres cis, trans, lesbianas (CTL) y el acceso a la justicia}

Es necesario entender la peligrosidad a la que están expuestas las mujeres CLT cuando se acentúan los obstáculos del acceso a la justicia, particularmente cuando se trata de casos de violencia de género. La situación empeora a medida que agregamos capas de vulnerabilidad, como el hecho de tener familiares a cargo, sufrir violencia económica (trabajo informal), vivir en situación de pobreza, no estar inserta en el mercado laboral, vivir en zonas alejadas, entre otras. Los obstáculos materiales, formales y simbólicos se traducen en infraestructura e información que no llega, en agentes que discriminan y re-victimizan, en protocolos que no existen o que no se aplican por falta de presupuesto, que impactan directamente en las vidas de estas niñas, adolescentes y mujeres. 
A partir de la reforma en el código penal, empezaron a nombrarse los crímenes padecidos por la población de mujeres trans y travestis. El travesticidio/transfemicidio es la expresión más visible y final de una cadena de violencias estructurales que responden a un sistema cultural, social, político y económico vertebrado por la división binaria excluyente entre los géneros. (Radí, Sardá-Chandiramani, 2016). Sin embargo, la resistencia de la justicia se impone al momento de aplicar tipologías penales invisibilizando crímenes que siguen siendo caratulados como homicidios simples o calificados, incluso sin respetar el nombre de pila de la víctima según su género auto percibido como establece la Ley de Identidad de Género (Ley 26.743, 2012). De esta manera, se omite la gravedad de los mismos, que son el resultado final de una serie de violencias estructurales por las que suele estar marcada la vida de las personas que integran el colectivo de mujeres trans y travestis. Entonces lo que la justicia no nombra configura un gran obstáculo en el acceso a la justicia, porque lo que no se nombra, no existe, no se registra y desaparece.

En cuanto al análisis de la migración, al iniciar el proceso migratorio la mujer atraviesa por múltiples escenarios desde su origen, tránsito, destino y el retorno; en lo que refiere a la salida de su país de origen primero porque si migran solas están sujetas a una mayor sanción social por parte de sus familias y comunidades, donde son consideradas malas madres y malas hijas que abandonan a sus familias, incluso haciéndolas responsables de cualquier dificultad que surja en el grupo familiar; como así también si se queda en su país de origen debiendo soportar sobrecarga laboral. Durante el tránsito, estudios plantean que es en esta etapa en la que más riesgos implica para la vida y la seguridad de las personas migrantes, especialmente para las mujeres, recurren en ocasiones al uso de documentos falsos, o se exponen a riesgos como ser la violencia sexual y la trata de personas.

En el caso de las mujeres indígenas se puede analizar las tres dimensiones de acceso a la justicia y sus respectivos obstáculos: 1) por un lado el sentido simbólico se refiere a las concepciones histórico culturales que se vinculan con el aun persistente proceso de colonización y colonialidad que nos atraviesa (Lugones, 2008), esto se materializa por ejemplo en la negación de la identidad indígena por parte del Estado, generando una descredibilidad por parte de las mujeres en el sistema de justicia; 2) el acceso en sentido material, está vinculado con las condiciones socio estructurales tales como las distancias geográficas, dificultades económicas, costos de acceso a los servicios jurídicos, pocas oportunidades para acceder al mercado laboral, a los servicios de salud y educación, acceso limitado a programas y servicios sociales, tasas elevadas de analfabetismo, escasa participación en el proceso político y marginación social; 3) en su dimensión formal o legal en el acceso a la justicia, las mujeres indígenas suelen encontrarse con otros obstáculos cuando se encuentra dentro del sistema de 
justicia donde el Estado debe garantizar el acceso a derechos como ser: proveer de intérpretes y traductores de manera gratuita cada vez que sean requeridas, contar a lo largo del proceso judicial con un cuerpo multidisciplinario de profesionales y personal capacitado que adopte un enfoque intercultural e interseccional, que respete la identidad cultural y étnica, el idioma y las características particulares de las mujeres indígenas.

Hemos de destacar, que más allá de las leyes en protección a los pueblos indígenas es preciso incorporar no sólo una perspectiva de género sino también intercultural e interseccional, para prevenir, investigar, enjuiciar y sancionar todas las formas de violencia contra las mujeres indígenas; erradicar la discriminación institucional y promover la participación activa de las mujeres indígenas en los procesos de construcción de normas que involucren sus derechos.

\section{Metodología utilizada}

El trabajo de investigación presentado tiene un enfoque cualitativo, que supone la inmersión de quien investiga en las prácticas de sus actores sociales. El método es elegido. (Yuni y Urbano, 2003 y 2006).

El método que orientará y enmarcará el proyecto es la Investigación AcciónParticipativa, nacido en el auge de las luchas populares, la expansión de los movimientos sociales y la crítica de las Ciencias Sociales a los métodos clásicos de investigación por su supuesta neutralidad y apoliticidad; además de poner en cuestión para quién se investiga y para qué (Ander- Egg, 1983).

En el marco del contexto regional Jujuy-Tucumán, en base a la implicancia de la metodología elegida, nos permite pensar a la Investigación Acción Participativa como una forma de democratización y socialización del saber en un sentido emancipatorio, porque los sectores participantes van adquiriendo dominio y comprensión de los procesos sociales en los que están inmersos.

Sin dudas, dicha metodología de investigación tiene como fuente de inspiración y conocimiento la pedagogía de Paulo Freire, que desde sus inicios buscó su coherencia en el intento de constituirse desde y con los oprimidos, antes que para ellos; y que por sobre todo se construye con la mentalidad puesta en la situación latinoamericana y en función de su problemática más aguda (Ander-Egg, 2003).

A su vez guiamos el proceso de Investigación Participativa por los diseños metodológicos propuestos desde los feminismos decoloniales. Estas metodologías proponen que la recuperación de los puntos de vista de las mujeres sea clave debido a que el androcentrismo de las ciencias y disciplinas lo han excluído. (Guerra Pérez, 2018). 


\subsection{Técnicas de recolección de datos}

Como hicimos referencia en el punto "2. Objetivos", las particularidades de las técnicas utilizadas en este proyecto, las hacen merecedoras de una explicación más profunda.

Una de las técnicas utilizadas, y quizás más innovadora es la historia de vida. La misma, es una estrategia de la investigación, encaminada a generar versiones alternativas de la historia social, a partir de la reconstrucción de las experiencias personales.

Esta técnica permite traducir lo cotidiano en palabras, gestos, símbolos, anécdotas, relatos, y constituye una expresión de la permanente interacción entre la historia personal y la historia social (Puyana y Barreto, 2010). La historia de vida proporciona una lectura de lo social a través del lenguaje, en el cual se expresan los pensamientos, y los deseos.

Otra técnica, utilizada de manera complementaria para recolectar datos, es la encuesta ya que permitió profundizar la información sobre las situaciones de vulneración de derechos en su organización y/o comunidad, y los conocimientos teórico-prácticos de las personas vulneradas. En su implementación el interés no radica en el sujeto concreto que contesta el cuestionario, sino la población a la que pertenece. Sierra Bravo (1999) lo define como un conjunto de preguntas, preparado cuidadosamente, sobre los hechos y aspectos relevantes en una investigación. A través de él, las encuestadas, previa su lectura, contestaron por escrito, sin intervención directa de persona alguna de las que forman parte de la investigación.

También utilizamos el mapeo participativo, modalidad que permitió registrar en forma gráfica participativa, los diferentes componentes de un lugar en estudio, permitiendo ubicarlos y describirlos en el espacio y en el tiempo, así como también documentar las percepciones que las personas tienen sobre el estado, su distribución y manejo (Ardón, M. 1998). Tiene como fundamentos conceptuales la investigaciónacción-participativa basada en el territorio como elemento fundamental de la metodología. La comunidad es partícipe de la investigación, aporta sus conocimientos y experiencia, de esta manera la construcción colectiva de mapas permite la reactualización de la memoria individual y colectiva.

Por último, como metodología innovadora, se realizaron talleres sincrónicos en modalidad virtual, como otra herramienta de recolección de dato; centrando la mirada en conocer sus experiencias de participación y ámbitos en los que se desenvuelven; recolectar datos para analizar la realidad socioeconómica, los recursos institucionales cercanos; conocer sus opiniones, percepciones e imaginarios en tornos a las violencias y el acceso a derechos de mujeres CTL; identificar 
problemáticas, poniendo foco en los obstáculos de acceso a la justicia; y fortalecer el rol de cada una en sus territorios y organizaciones.

\section{Datos}

Como nuestro objetivo general, es identificar los obstáculos de acceso a la justicia de las mujeres CTL de las provincias de Jujuy y Tucumán en situación de violencia, estamos en condiciones de presentar algunos de los datos que arrojó nuestro primer año de investigación (2020- De investigación participativa):

En primer lugar, en cuanto a las características grupales, las mujeres CTL referentes de organizaciones de ambas provincias. pertenecientes a diferentes sectores:

- $\quad$ Se encuentran en el grupo etáreo de entre 19 y 58 años.

- La mayoría (80\%) cuenta con estudios primarios y secundarios completos, y en porcentajes menores, lograron finalizar el nivel terciario y/o universitario.

Sus espacios de trabajo mayormente se caracterizan por la informalidad. De esta manera, la pandemia tuvo un gran impacto, profundizando las desigualdades ya existentes.

- $\quad$ Casi el total (90\%) de las mujeres, participaron y actualmente forman parte de una organización, manifestando que formar parte de un grupo, les permite constituir una red de acompañamiento, ayuda y apoyo, como así también en términos identitarios.

- Manifiestan como lineamiento axial que orienta sus prácticas y experiencias de participación, es proteger, cuidar y custodiar los derechos de Mujeres, Niños, Niñas y Adolescentes.

Entre las formas de violencia y situaciones de violencias que manifiestan haber sufrido, se destacan la:

-Violencia institucional, violencia psicológica y violencia simbólica.

-En estrecha relación, respecto a situaciones de discriminación, el principal porcentaje -en el caso de Tucumán- es por motivos de género a diferenca de Jujuy, donde lo racial adquiere centralidad.

Respecto a los obstáculos de acceso a la justicia y tomando como punto de partida que la mayoría de las participantes expresa haber sufrido situaciones de violencia y/o haber presenciado/conocido personas que atraviesan por esto, los mayores obstáculos de acceso a la justicia, en las provincias de Jujuy y Tucumán son: 


\section{Obstáculos Formales}

- $\quad$ Falencias e ineficiencias en los circuitos de denuncia para las víctimas de violencia de género. Como resultado de la excesiva burocratización de las vías de resolución, de los circuitos interminables y de las respuestas lentas y poco integrales, las víctimas de violencia terminan alejándose de los sistemas judiciales.

Malas prácticas de las instituciones públicas, relacionadas con la falta de capacitación en perspectiva de género del personal que allí trabaja. A su vez, esto se encuentra relacionado con la falta de voluntad política de los Estados provinciales y municipales a la hora de aplicar la Ley Micaela de forma obligatoria. Como así también la falta de una perspectiva intercultural en las oficinas estatales como en las políticas públicas que se llevan adelante.

En cuanto al colectivo de mujeres travestis y trans, prevalece la desconfianza y el alejamiento del Estado y sus instituciones, ya que se sienten expuestas ante el destrato y la discriminación que ataca e invisibiliza sus identidades.

- La despersonalización, la deshumanización y la falta de empatía en la asistencia de los agentes estatales.

- Las mujeres indígenas y migrantes ven vulnerado su derecho de acceder a la justicia por la falta de traductores plurilingües.

- El Incumplimiento de medidas judiciales de acercamiento, Incumplimiento de los protocolos de investigación en casos de femicidios, travesticidios y transfemicidios.

Los Obstáculos vinculados con las instituciones públicas, sus procedimientos y actuaciones haciendo hincapié en la actuación policial. Las falencias en la actuación de las fuerzas de seguridad están centradas en la inacción, la falta de conocimiento en perspectiva de género, desconocimiento de los protocolos, incumplimiento de sus funciones como los retrasos en la toma de denuncia, la revictimización, la falta de respuesta a la hora de ejecutar medidas dictadas por la justicia, el maltrato y la discriminación en la asistencia a vícitmas.

Además, existe un abuso de poder de parte de los policías como funcionarios públicos y no existen entidades que controlen el accionar ni establezcan sanciones justas.

Obstáculos Simbólicos

- Las practicas sociales que naturalizan la desigualdad y la discriminación, distinguidos sobretodo, en aquellos que se vinculan a la hetero-cis norma.

- El cuestionamiento en las decisiones que toma una mujer en situación de violencia. 
- Acuerdos y encubrimiento de los agresores, femicidas y transfemicidas.

Los medios de comunicación, generando discursos en contra de las personas pobres o adictas.

- La reproducción de discursos sociales negativos sobre ciertos grupos, mujeres, personas trans, que desvían las responsabilidades estatales.

- Una sociedad con principios machistas, patriarcales y desiguales, que discrimina a las mujeres, a las disidencias y mucho más si pertenecen a comunidades indígenas, migrantes y sectores populares.

La discriminación racial y cultural que atraviesan las mujeres indigenas a causa de un Estado que no dialoga con las comunidades y a causa de una sociedad racista.

Obstáculos Materiales

- $\quad$ El poco entendimiento de las víctimas del proceso judicial, su lenguaje y sus circuitos.

- $\quad$ Ante la situación de una mayor desigualdad económica, la ausencia del Estado incapaz de garantizar el derecho a la vivienda y a un trabajo digno.

- La falta de educación en general, el analfabetismo y específicamente la desinformación arraigada a la educación sin perspectiva de género y de derechos. Lo cual marcan que se visualiza en el sistema educativo formal. Falta de Educación Sexual Integral.

- Las mujeres indígenas se ven fuertemente afectadas por las distancias geográficas a causa que sus comunidades no están ubicadas en los centros urbanos, viendo afectado su acceso debido a la centralización de las acciones de las instituciones, en las ciudades cabeceras de los departamentos de Jujuy y Tucumán. Esto dificulta y vuelve casi imposible concretar acciones para poder atender las situaciones de violencias.

Los conflictos ligados al derecho al territorio y a los desalojos que vienen enfrentando las comunidades a lo largo del tiempo, con fuertes represiones y donde las mujeres son las ponen el cuerpo.

\section{Reflexiones del trabajo}

Podemos decir que las beneficiarias del proyecto identifican y reconocen derechos vulnerados, como así también la comprensión del proceso de evolución de los mismos y la necesidad de pensarlos a partir de acciones que los hacen más específicos en pos de proteger a grupos vulnerados. Es por ello, que declaran la existencia de logros y avances en materia de derechos y ponen énfasis que estos están 
en permanente interpelación, reivindicando las luchas de los movimientos feministas y LGTBIQP+, como generadores de los mismos y sobretodo actores que garantizan su efectivización.

No obstante, estas valoraciones entran en tensión al hacer un análisis crítico del impacto real y la efectivización de las políticas públicas; marcando un estancamiento en las capacidades reales del Estado de llevar a cabo acciones que garanticen y aseguren los derechos. Estas fallas, no sólo son registradas por parte de las instituciones estatales (policial, judiciales, educativas, etc), sino que se reconocen que vienen enraizadas a una estructura patriarcal y desde una lógica hegemónica colonial.

De este modo, se podría decir, que se comprueba que ha habido un avance significativo en las condiciones estructurales en materia de avance de derechos, legislación, estrategia y políticas públicas, sin embargo puede observarse un estancamiento en las capacidades estatales e institucionales. Utilizando la guía de indicadores de progreso para la medición de la implementación de la Convención de Belém do Pará (2015) se puede decir, que existe "una deficiencia de las acciones implementadas por el Estado para asegurar un avance en la garantía de los derechos pese a que puedan estar dadas las condiciones estructurales para hacerlo”.

\section{Conclusión}

La presente publicación, tiene como fin presentar el proyecto de investigación participativa que estamos llevando, a nivel regional, tanto en Jujuy como en Tucumán. No solo por su importante estructura, sino también porque entendemos que la visibilización y sensibilización de manera concreta permite generar conciencia, y así desarrollar colectivamente prácticas reparadoras.

Queremos contribuir, como organización, a la erradicación de la violencia de género de las mujeres CTL, aportando datos reales sobre los obstáculos de acceso a la justicia de las mismas; y generando procesos de emancipación y empoderamiento en las mujeres beneficiarias del mismo, porque como organización entendemos a los derechos humanos como una herramienta de cambio social.

Es por ello, que el proyecto se lleva a cabo en construcción colaborativa y participativa con quienes resultan vulnerables ante las posibilidades de acceso a la justicia, ya que como plantean varios autores, la justicia solo existe si nadie queda afuera, de lo contrario no hay justicia.

Esperamos despertar inquietudes en el resto de las provincias, y contagiar esta forma de investigación, en la que no solo entramos en conocimiento de las problemáticas existentes, sino que además, se involucra a las personas afectadas y que 
realizan trabajos territoriales, a los fines de arribar a una solución efectiva y satisfactoria.

\section{Referencias bibliográficas}

ANDER EGG, Ezequiel. (1983). Técnicas de investigación social. Buenos Aires: El Cid Editor.

ARDÓN MEJÍA, Mario. (1998) Serie de Cuadernos Metodológicos de Investigación Participativa (seis cuadernos). Zamorano, Tegucigalpa.

ARROYO VARGAS, Roxana (2012) Acceso a la justicia para las mujeres... El laberinto androcéntrico del derecho de Umbral revista de derecho constitucional. Centro de Estudios y Difusión del Derecho Constitucional. -Semestral. -- Quito. -- No. 2 http://www.corteidh.or.cr/tablas/r26673.pdf

ARROYO VARGAS, Roxana (2001), La igualdad un largo camino para las mujeres, parte del trabajo Reflexiones para una defensa de tesis doctoral. Madrid, España. http://www.facilitar.io/sites/default/files/resources/documents/2017-

12/ARROYO\%20VARGAS_Igualdad\%20un\%20largo\%20camino\%20para\%201 as\%20mujeres.pdf

BIRGIN, Haydée (2012). Acceso a la justicia y violencias: Una deuda con los derechos de las mujeres. En La Justicia en Construcción. ELA.

CAMUÑA, Pablo (2005) El Derecho como herramienta para un cambio social basado en la igualdad y la inclusión, ANDHES.

CHAVES GROH, María José; ZAMORA UREÑA, Juan Carlos (2014) Organización Internacional para las Migraciones. Módulo sensibilizandonos sobre las realidades de las mujeres migrantes. San José, C.R.: OIM, Estados Unidos. Depto. de Estado. Oficina de Población, Refugiados y Migración.

DE MIGUEL DÍAZ, Francisco Mario. (1989). Modelos de investigación sobre organizaciones educativas, Revista de Investigación Educativa, 7 (13), 21-56.

ELLIOTT, John. (2000). El cambio educativo desde la investigación-acción, Madrid: Morata.

ESPINOSA-MIÑOSO, Yuderkys (2014). Una crítica descolonial a la epistemología feminista crítica. Ed.: El Cotidiano 184

GONZÁLEZ FARRERAS, Julia y SETIÉN, María Luisa (2005) Diversidad Migratoria, distintos protagonistas, diferentes contextos. Universidad de Deusto Bilbao.

GUERRA PÉREZ, Mariana Noel (2018). Notas para una investigación feminista decolonial. Vinculaciones epistemológicas. RELIGACIÓN-Revista de Ciencias Sociales y Humanidades, Vol. 3, No 9. 
KAUFMAN, Esther (1991) El ritual jurídico en el juicio a los ex comandantes. La desnaturalización de lo cotidiano, en Guber, Rosana, El salvaje metropolitano, Buenos Aires, Legasa.

LOVATÓN PALACIOS, Palacios, David (2007) Acceso a la justicia: llave para la gobernabilidad democrática. Washington: Organización de los Estados Americanos - Consorcio Justicia Viva.

LUGONES, María (2008) Colonialidad y Género. Binghamton University, USA. TABULA RASA. No.9

MARABOTTO LUGARO, Jorge A (2003). Un derecho Humano Esencial: el Acceso a la Justicia. Biblioteca Jurídica Virtual del Instituto de Investigaciones Jurídicas de la

UNAM. http://historico.juridicas.unam.mx/publica/rev/indice.htm?r $=$ dconstla\&n=20 03

MARTÍNEZ, Miguel. (2006). La investigación cualitativa (Síntesis Conceptual), en Revista IPSI, Volumen 9, Número 1, Facultad de Psicología, Caracas: Universidad Simón Bolívar.

MCKERNAN, James. (1999). Investigación-acción y curriculum. Madrid: Morata. RODRÍGUEZ GÓMEZ, Gregorio; GIL FLORES, Javier. y GARCÍA JIMÉNEZ, Eduardo. (1996). Metodología de la Investigación Cualitativa. Granada. Aljibe.

RUIZ, Alicia E. C. (2008) Vulnerabilidad y Violencia. Defensa Pública: garantía de acceso a la justicia, Ministerio Público de la Defensa. La Ley.

SANDÍN, Esteban, Ma P. (2003). Investigación Cualitativa en Educación. Fundamentos y Tradiciones. Madrid. McGraw Hill.

SIERRA BRAVO, Restituto. (1999). Técnicas de investigación Social. Teoría y Ejercicios. Paraninfo. España.

STOLCKE, Verena (2000) ¿Es el sexo para el género lo que la raza para la etnicidad...y la naturaleza para la sociedad?. Política y Cultura. Red de revistas científicas https://www.redalyc.org/articulo.oa?id=267/26701403

PERELLÓ OLIVER, Salvador (2009). Metodología de la Investigación Social. Madrid: Dykinson.

PIQUÉ, María Luisa. PZELLINSKY, Romina (2014) Obstáculos en el acceso a la justicia de mujeres víctimas de violencia de género. Revista jurídica: Universidad de

Palermo https://www.palermo.edu/derecho/revista_juridica/pub14/Revista_Juridica_Ano14-N2_10.pdf

PUYANA VILLAMiZAR, Yolanda. \& BARRETO GAMA Juanita. (2010). La historia de vida: Recurso en la investigación cualitativa Reflexiones metodológicas. Maguaré 
VIEYTES, Rut. (2004). Metodología de la investigación en organizaciones, mercado y sociedad: epistemología y técnicas. Buenos Aires: Edito.

YUNI, José Alberto - URBANO, Claudio Ariel (2003). Técnicas para investigar y formular proyectos de investigación. Volumen I. Córdoba: Brujas.

YUNI, José Alberto - URBANO, Claudio Ariel (2006). Técnicas para investigar. Recursos metodológicos para la preparación de proyectos de investigación. Brujas

\section{Otras Fuentes:}

Convención de Belém Do Pará (1996).

Informe Anual del Comité Jurídico Interamericano a la Asamblea General (2008), Comité Jurídico Internacional.

Informe El acceso a la Justicia como garantía de Los Derechos Económicos, Sociales y Culturales. Estudio de los estándares fijados por el Sistema Interamericano de Derechos Humanos, de la Comisión Interamericana de Derechos Humanos (CIDH) http://www.cidh.oas.org/countryrep/AccesoDESC07sp/Accesodesciii.sp.htm\#La\%20obligaci\%C3\%B3n\%20de\%20proveer\%20servicios

Informe Obstáculos para el Acceso a la Justicia en las Américas (2009), Instituto de Defensa Legal de Perú y Fundación del Debido Proceso Legal.

Informe Panorama de las mujeres en América Latina y el Caribe en contextos económicos inestables (2019), Fundación para Estudio e Investigación de la Mujer (FEIM).

Ley 26.485 de Protección Integral a las Mujeres (2009).

Programa de las Naciones Unidas para el Desarrollo, Manual de políticas públicas para el acceso a la justicia (2005), Instituto Talcahuano, Buenos Aires.

Reglas de Brasilia para el Acceso a la Justicia de Personas en Condición de Vulnerabilidad, (2008).

Resumen gráfico de las principales ideas y conceptos del informe: "Las Mujeres Indígenas y sus Derechos Humanos en las Américas” (2017), de la Comisión Interamericana de Derechos Humanos (CIDH)

https://www.oas.org/es/cidh/indigenas/docs/pdf/Brochure-MujeresIndigenas.pdf 